\title{
Zonisamide enhances neurite outgrowth from adult rat dorsal root ganglion neurons, but not proliferation or migration of Schwann cells
}

\author{
Shizuka Takaku ${ }^{1}$ Kazunori Sango ${ }^{1}$ \\ Accepted: 16 December 2019 / Published online: 26 December 2019 \\ (c) The Author(s) 2019
}

\begin{abstract}
Zonisamide, an anti-epileptic and anti-Parkinson's disease drug, displays neurotrophic activity on cultured motor neurons and facilitates axonal regeneration after peripheral nerve injury in mice, but its underlying mechanisms remain unclear. In this study, zonisamide enhanced neurite outgrowth from cultured adult rat dorsal root ganglion (DRG) neurons in a concentrationdependent manner $(1 \mu \mathrm{M}<10 \mu \mathrm{M}<100 \mu \mathrm{M})$, and its activity was significantly attenuated by co-treatment with a phosphatidyl inositol-3'-phosphate-kinase (PI3K) inhibitor LY294002 or a mitogen-activated protein kinase (MAPK) inhibitor U0126. In agreement with these findings, $100 \mu \mathrm{M}$ zonisamide for $1 \mathrm{~h}$ induced phosphorylation of AKT and ERK1/2, key molecules of PI3K and MAPK signaling pathways, respectively in mouse neuroblastoma $\times$ rat DRG neuron hybrid cells ND7/23. In contrast, zonisamide failed to promote proliferation or migration of immortalized Fischer rat Schwann cells 1 (IFRS1). These findings suggest that the beneficial effects of zonisamide on peripheral nerve regeneration may be attributable to its direct actions on neurons through PI3K and MAPK pathways, rather than the stimulation of Schwann cells.
\end{abstract}

Keywords Zonisamide - Dorsal root ganglion neurons · Neurite outgrowth · ND7/23 cells · Signaling pathways · Immortalized Schwann cells

\section{Introduction}

Successful axonal regeneration in the peripheral nervous system (PNS) depends on the capacity of neurons and/or Schwann cells to regenerate neurites, the environment distal to the injury, and the target tissues receptive to reinnervation (Sango et al. 2017). The sequence of cellular events during Wallerian degeneration includes Schwann cell activation and proliferation, macrophage recruitment, elimination of axonal and myelin debris, and synthesis of neurotrophic and chemotactic factors. However, axonal regeneration and reinnervation depend on appropriate contact of regenerating axonal sprouts with Schwann cell basal laminae in the distal nerve segment. Clinical approaches to repair axonal injury are still far from satisfactory, and no effective drugs are available to

Kazunori Sango

sango-kz@igakuken.or.jp

1 Diabetic Neuropathy Project, Department of Sensory and Motor Systems, Tokyo Metropolitan Institute of Medical Science, 2-1-6 Kamikitazawa, Setagaya-ku, Tokyo 156-8506, Japan promote axonal regeneration with functional restoration after injury (Faroni et al. 2015).

Drug repositioning or repurposing is a strategy to discover new efficacies of drugs currently applicable to patients, and may significantly diminish the cost and time for developing drugs as compared with conventional approaches (Padhy and Gupta 2011). Zonisamide, a benzisoxazole derivative, was initially developed as an anti-epileptic drug (Jain 2000), and has been successfully repositioned for Parkinson's disease (Murata et al. 2015; Murata et al. 2016). Yagi et al. (2015) found that zonisamide promoted neurite outgrowth from cultured motor neurons and facilitated axonal regeneration after sciatic nerve injury in mice. These findings provide evidence of neurotrophic and neuroprotective properties of zonisamide in the PNS and suggest its potential repositioning for peripheral nerve injury; however, the underlying mechanisms remain unclear. By employing primary-cultured adult rodent sensory neurons of dorsal root ganglia (DRGs) and immortalized Fischer rat Schwann cells 1 (IFRS1) (Sango et al. 2011), we investigated the functional roles of numerous growth factors and cytokines in peripheral nerve degeneration and regeneration (Sango et al. 2008; Takaku et al. 2013; Tsukamoto et al. 2015a, b). The aim of the present study is 
to elucidate the potential efficacy of zonisamide for neurite outgrowth from DRG neurons and proliferation/migration of IFRS1. In addition, neuroblastoma $\times$ DRG neuron hybrid ND7/23 cells (Wood et al. 1990) were employed for investigating the signaling molecules and pathways mediating the neurite outgrowth-promoting effects of zonisamide.

\section{Materials and methods}

\section{Primary culture of adult rat DRG neurons}

Three-month-old female Wistar rats were obtained from Japan Clea (Shizuoka, Japan). All the experiments were conducted in accordance with the Guideline for the Care and Use of Animals (Tokyo Metropolitan Institute of Medical Science 2011). Dissociated cell culture of DRG neurons was performed as previously described (Takaku et al. 2013). Briefly, DRGs from the cervical to the lumbar level were dissected from each animal and dissociated with collagenase (CLS-3; Worthington Biochemicals, Freehold, NJ, USA) and trypsin (Sigma, St. Louis, MO, USA). These ganglia were subjected to density gradient centrifugation $(5 \mathrm{~min}$, $200 g$ ) with $30 \%$ Percoll PLUS ${ }^{\mathrm{TM}}$ (GE Healthcare Bio-Sciences Corp., Piscataway, NJ, USA) to eliminate the myelin sheath. This procedure resulted in a yield of more than $5 \times 10^{4}$ neurons along with a smaller number of Schwann cells and fibroblasts.

\section{Neurite outgrowth assay}

The dissociated DRG neurons were suspended in Dulbecco's Modified Eagle's medium (DMEM; Thermo Fisher Scientific Inc., Waltham, MA, USA) with 10\% fetal bovine serum (FBS; Thermo Fisher) and seeded onto polyL-lysine (PL; Sigma, $10 \mu \mathrm{g} / \mathrm{mL}$ )-coated wells of 8-well chamber slides (Nalge Nunc International, Naperville, IL, USA). The density of neurons was adjusted to approximately $2 \times 10^{3} / \mathrm{cm}^{2}$ in each well. After remaining in the serum-containing medium for $16 \mathrm{~h}$, the cells were cultured for $32 \mathrm{~h}$ in DMEM with serum-free medium supplement B27 (Thermo Fisher) and different concentrations (0, 1, 10 , or $100 \mu \mathrm{M}$ ) of zonisamide. Then the neurons were fixed with acid alcohol (95\% ethanol and 5\% acetic acid) at $4{ }^{\circ} \mathrm{C}$ for $10 \mathrm{~min}$, incubated overnight at $4{ }^{\circ} \mathrm{C}$ with the mouse anti- $\beta$ III tubulin monoclonal antibody (1:2000; Sigma) and then incubated for $1 \mathrm{~h}$ at $37{ }^{\circ} \mathrm{C}$ with the peroxidaseconjugated anti-mouse IgG antibody (1:200; MBL Corp., Ltd., Nagoya, Japan). Immunoreactions were visualized under a light microscope with $0.01 \%$ diaminobenzidine tetrahydrochloride (Wako Co., Tokyo, Japan) and $0.01 \%$ hydrogen peroxide (Sigma) in $50 \mathrm{mM}$ Tris buffer ( $\mathrm{pH}$ 7.4) at $37^{\circ} \mathrm{C}$ for $10 \mathrm{~min}$. The average neurite length (in $\mu \mathrm{m}$ ) was calculated from the measurements of approximately 700 neurites from 5 different cultures of each experimental group using digital images (MetaMorph System, Molecular Devices, Inc., Sunnyvale, CA, USA), and was normalized as the relative value of the control group.

To investigate the signaling pathways mediating the promoting effects of zonisamide on neurite outgrowth, $10 \mu \mathrm{M}$ of a phosphatidylinositol-3'-phosphate-kinase (PI3K) inhibitor LY294002 (Cell Signaling Technology, Beverly, MA, USA) or a mitogen-activated protein kinase (MAPK) kinase (MEK) inhibitor U0126 (Calbiochem; EMD Chemicals, Inc., San Diego, CA, USA) were cotreated with $100 \mu \mathrm{M}$ zonisamide.

\section{Culture of ND7/23 cells and western blotting}

Mouse neuroblastoma/rat embryonic DRG neuron hybrid ND7/23 cells (Wood et al. 1990) were kindly provided by Prof. Atsufumi Kawabata and Dr. Fumiko Sekiguchi, Laboratory of Pharmacology and Pathophysiology, Faculty of Pharmacy, Kindai University, Higashi-Osaka, Japan (Mitani et al. 2016). The cells at the passage of 15-20 were maintained in DMEM supplemented with 5\% FBS, and employed for western blot analysis (Takaku et al. 2013). The cells at semi-confluency in $60 \mathrm{~mm}$ culture plates were incubated in DMEM/B27 with $100 \mu \mathrm{M}$ zonisamide for 0 , $5,10,30,60$ and $120 \mathrm{~min}$, and lysed with $2 \times$ sodium dodecyl sulfate (SDS) sample buffer. SDS-polyacrylamide gel electrophoresis (SDS-PAGE) was performed using 10\% SDS-PAGE gel (Fujifilm, Tokyo, Japan). After electrophoresis, the protein was transferred onto a PVDF membrane with an electroblotter (Nihon Eido Co., Ltd., Tokyo, Japan). The membrane was incubated in Tris-buffered saline with $0.1 \%$ Tween 20 (including 5\% skimmed milk or $5 \%$ bovine serum albumin) for $1 \mathrm{~h}$ at room temperature, and then overnight at $4{ }^{\circ} \mathrm{C}$ with the following antibodies:

1. anti-AKT antibody (1:1000; Cell Signaling);

2. anti-phospho-AKT antibody (1:1000; Cell Signaling);

3. anti-extracellular signal-regulated kinase (ERK) $1 / 2$ antibody (1:1000; Cell Signaling);

4. anti-phospho-ERK1/2 (pT202/pY204) antibody (1:2000; Cell Signaling).

5. After rinsing with PBS containing $0.1 \%$ Tween 20 , the membrane was incubated in a solution of HRP-conjugated anti-rabbit IgG antibody or anti-mouse IgG antibody (1:2000; MBL) for $1 \mathrm{~h}$. After rinsing, immunocomplexes on the membrane were visualized with ECL plus a Western Lighting Ultra (PerkinElmer, Inc., Waltham, MA, USA). 


\section{Culture of IFRS1 and assays for proliferation/ survival and migration}

Spontaneously immortalized Fischer rat Schwann cells 1 (IFRS1) from adult Fischer 344 rats (Sango et al. 2011) at the passage of 30-40 were maintained in DMEM supplemented with 5\% FBS, and employed for the following assays.

The effects of zonisamide on the proliferation/survival of IFRS1 were evaluated using the CellTiter $96^{\circledR}$ AQueous One Solution Cell Proliferation Assay kit (Promega, Madison, WI, USA) according to the manufacturer's instructions (Tsukamoto et al. 2015b). The cells were seeded onto each well of 96-well culture plates at an approximate density of $3 \times 10^{4} / \mathrm{cm}^{2}$, and were incubated overnight in DMEM supplemented with $5 \%$ FBS. The cells were then maintained in DMEM/1\% FBS with different concentrations $(0,1,10$, or $100 \mu \mathrm{M})$ of zonisamide or $2 \mu \mathrm{M}$ forskolin as a positive control (Yamada et al. 1995; Sango et al. 2011) for 1 day and 3 days. After rinsing with $250 \mu \mathrm{L}$ of DMEM, the cells were incubated for $1 \mathrm{~h}$ at $37^{\circ} \mathrm{C}$ in $100 \mu \mathrm{L}$ of DMEM with $10 \mu \mathrm{L}$ of CellTiter $96^{\circledR}$ AQueous One Solution Reagent, and absorbance at $490 \mathrm{~nm}$ was determined with a microplate reader (Varioskan Flash; Thermo Fischer).

The effects of zonisamide on IFRS1 cell migration were evaluated by the scratch wound assay (Liang et al. 2007; Roloff et al. 2013). Briefly, the cells were seeded onto a PLcoated $35 \mathrm{~mm}$ glass-bottomed dish with grid (Matsunami Glass Ind., LTD, Osaka, Japan) at an approximate density of $2 \times 10^{4} / \mathrm{cm}^{2}$, and maintained in DMEM/5\%FBS for $24 \mathrm{~h}$. Then a cell-free area was scratched using a sterile $200 \mu \mathrm{L}$ pipette tip (BM Equipment Co., Ltd, Tokyo, Japan). The cells were incubated in DMEM/1\%FBS in the presence or absence of $100 \mu \mathrm{M}$ zonisamide, and the scratch was photographed $2 \mathrm{~h}$ (0 day) and 3 days after its generation using a phase-contrast microscope (IMT-2; Olympus, Tokyo, Japan) equipped with a microscope digital camera system (DP22-CU; Olympus) and image analysis software (WinROOF2015; Mitani Corporation, Tokyo, Japan). The number of cells migrating into the square area of scratch $(6 \times 6$ grids) was calculated by reducing the number in the area at 3 days from that at 0 day, and expressed as the relative value of the control condition.

\section{Statistical analysis}

All the data are expressed as means with SD, and the number of experiments is indicated in the figure legends. Parametric comparisons between experimental groups were performed by one-way analysis of variance (ANOVA); when ANOVA showed a significant difference between groups $(P<0.05)$,
Tukey-Kramer test was used to identify which group differences accounted for the significant $P$ value.

\section{Results and discussion}

\section{Zonisamide promotes neurite outgrowth from DRG neurons}

In the previous study (Yagi et al. 2015), zonisamide dosedependently $(1 \mu \mathrm{M}<10 \mu \mathrm{M}<20 \mu \mathrm{M})$ promoted neurite outgrowth from primary-cultured motor neurons. In agreement with that finding, we observed that treatment with zonisamide enhanced neurite outgrowth from DRG neurons in a concentration-dependent manner; zonisamide at $10 \mu \mathrm{M}$ and $100 \mu \mathrm{M}$ significantly increased the relative neurite length as compared with control (Fig. 1). In addition to the neurite length, we measured the diameters of neurons in the control and $100 \mu \mathrm{M}$ zonisamide-treated groups, respectively,

(a)

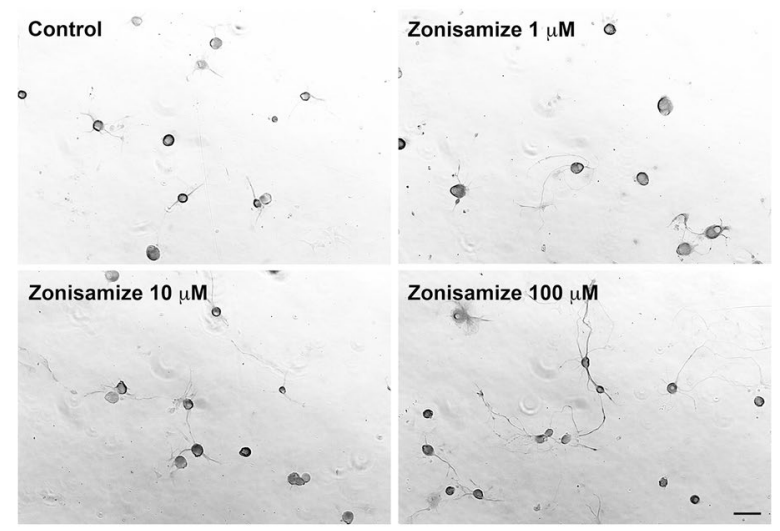

(b)

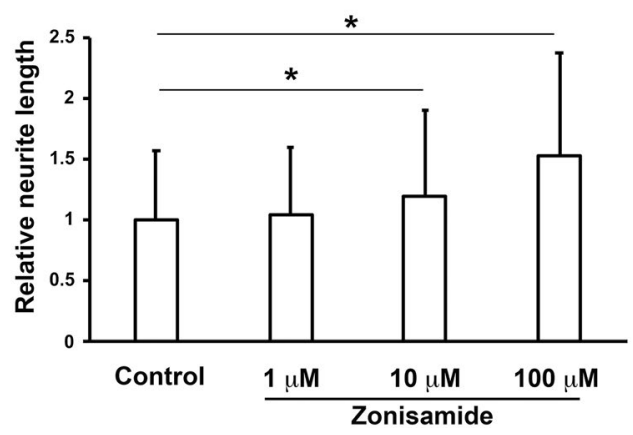

Fig. 1 Zonisamide promotes neurite outgrowth from adult rat DRG neurons after 2 days in culture. a Representative photomicrographs of control and zonisamide $(1 \mu \mathrm{M}, 10 \mu \mathrm{M}$, and $100 \mu \mathrm{M})$-treated DRG neurons immunostained with anti- $\beta$ III tubulin antibody. Scale bar $=50 \mu \mathrm{m}$. b Concentration-dependent effects of zonisamide on neurite outgrowth; bar charts of the relative neurite length in each culture condition. Values represent means + SD from 665 to 816 neurites; $* P<0.01$ 
and saw no significant differences in the average value between the former $(26.6 \pm 2.7 \mu \mathrm{m} ; n=90)$ and the latter $(26.1 \pm 2.6 \mu \mathrm{m} ; n=90)$. These findings suggest that zonisamide increases the neurite length without altering the size of neuronal cell bodies. Because the promoting effects of zonisamide were significantly attenuated by co-treatment with $10 \mu \mathrm{M}$ of a PI3K inhibitor LY294002 or a MEK inhibitor U0126 (Fig. 2), PI3K and MAPK signaling pathways appear to play a role in the zonisamide-induced promotion of neurite outgrowth. These findings are similar to those in our previous study (Sango et al. 2008), which described the inhibitory effects of LY294002 and U0126 on the neurite outgrowth-promoting activity of ciliary neurotrophic factor (CNTF). CNTF acts on neurons via the cell-surface receptor complex (CNTF receptor $\alpha$, gp130, and LIF receptor) to activate the signaling pathways, such as Janus kinase (JAK)/

(a)
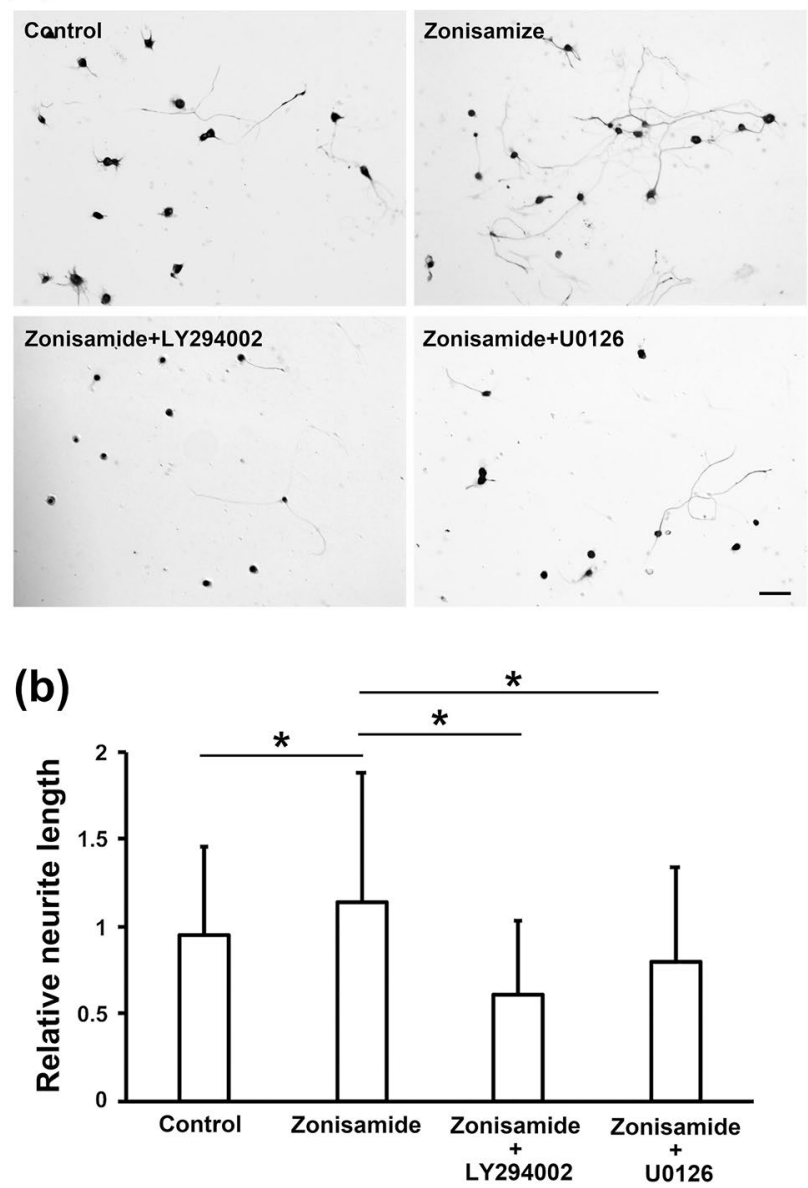

Fig. 2 The neurite outgrowth-promoting effects of $100 \mu \mathrm{M}$ zonisamide are attenuated by co-treatment with $10 \mu \mathrm{M}$ of LY294002 or U0126. a Representative photomicrographs of control, zonisamide-, zonisamide + LY294002-, and zonisamide + U0126-treated DRG neurons immunostained with anti- $\beta$ III tubulin antibody. Scale bar $=50 \mu \mathrm{m}$. b Bar charts of the relative neurite length in each culture condition. Values represent means + SD from 361 to 504 neurites; $* P<0.01$ signal transducer and activator of transcription 3 (STAT3), PI3K/AKT, and MEK/MAPK, whereas receptors for zonisamide have not been identified and the mechanisms of its neurotrophic activity remain largely unknown.

Next, we tried to look into phosphorylation of AKT and ERK1/2 in DRG neurons in the presence or absence of zonisamide; however, the amount of protein obtained from the primary-cultured DRG neurons was insufficient to evaluate the phosphorylated state of each molecule by western blotting. We then substituted them with DRG neuron $\times$ neuroblastoma hybrid cells ND7/23, which possess high proliferative activity with some characteristic features of nociceptive sensory neurons, such as expression of substance $\mathrm{P}$ and high-affinity neurotrophin receptor TrkA (Wood et al. 1990; Mitani et al. 2016; Yin et al. 2016). The western blot analysis revealed that $100 \mu \mathrm{M}$ zonisamide for $60 \mathrm{~min}$ or $120 \mathrm{~min}$ induced phosphorylation of AKT and ERK1/2 in ND7/23 cells (Fig. 3). Numerous kinds of neuroprotective molecules have been shown to enhance neurite outgrowth through the activation of PI3K/AKT and MEK/MAPK pathways (Okada et al. 2010; Zigmond 2012; Chan et al. 2014). In addition, Saijilafu et al. (2013) reported that PI3K pathway activated in response to axonal injury was required for sensory nerve regeneration. Although no direct evidence was provided, PI3K/AKT pathway might be involved in the neuroprotective function of zonisamide. For instance, zonisamideinduced up-regulation of manganese superoxide dismutase through the activation of PI3K pathway might contribute to the attenuation of 1-methyl-4-phenyl-1,2,3,6-tetrahydropyridine (MPTP)-induced apoptotic cell death of SH-SY5Y neuroblastoma cells (Kawajiri et al. 2010). According to the study by Yagi et al. (2015), zonisamide tended to suppress phosphorylation of ERK1/2 in primary-cultured motor neurons and NSC-34 motor neuron-like cells. The reasons for the opposite effects of zonisamide on ERK1/2 phosphorylation between ND7/23 cells and the motor neurons remain unknown, but one of plausible explanations might be the expression of TrkA receptor in the former, but not in the latter. MAPK signal transduction cascade plays a major role in the neurotrophic activity of nerve growth factor (NGF) and related molecules on TrkA-expressing DRG neurons and ND7/23 cells (Ulmann et al. 2009; Inoue et al. 2012), whereas NGF failed to promote the survival of TrkA-negative NSC-34 cells (Matusica et al. 2008). However, it needs further consideration on how zonisamide activates TrkAMAPK signaling pathway. It is also noteworthy that activation of ERK $1 / 2$ in neuronal cells is involved in not only the effects of neuroprotective molecules (Zigmond 2012), but also the negative events, e.g. neuronal cell death caused by oxidative stress (Satoh et al. 2000). The findings of our study suggest that zonisamide-induced ERK1/2 phosphorylation contributes to the promotion of neurite outgrowth in DRG neurons, whereas zonisamide might attenuate its 


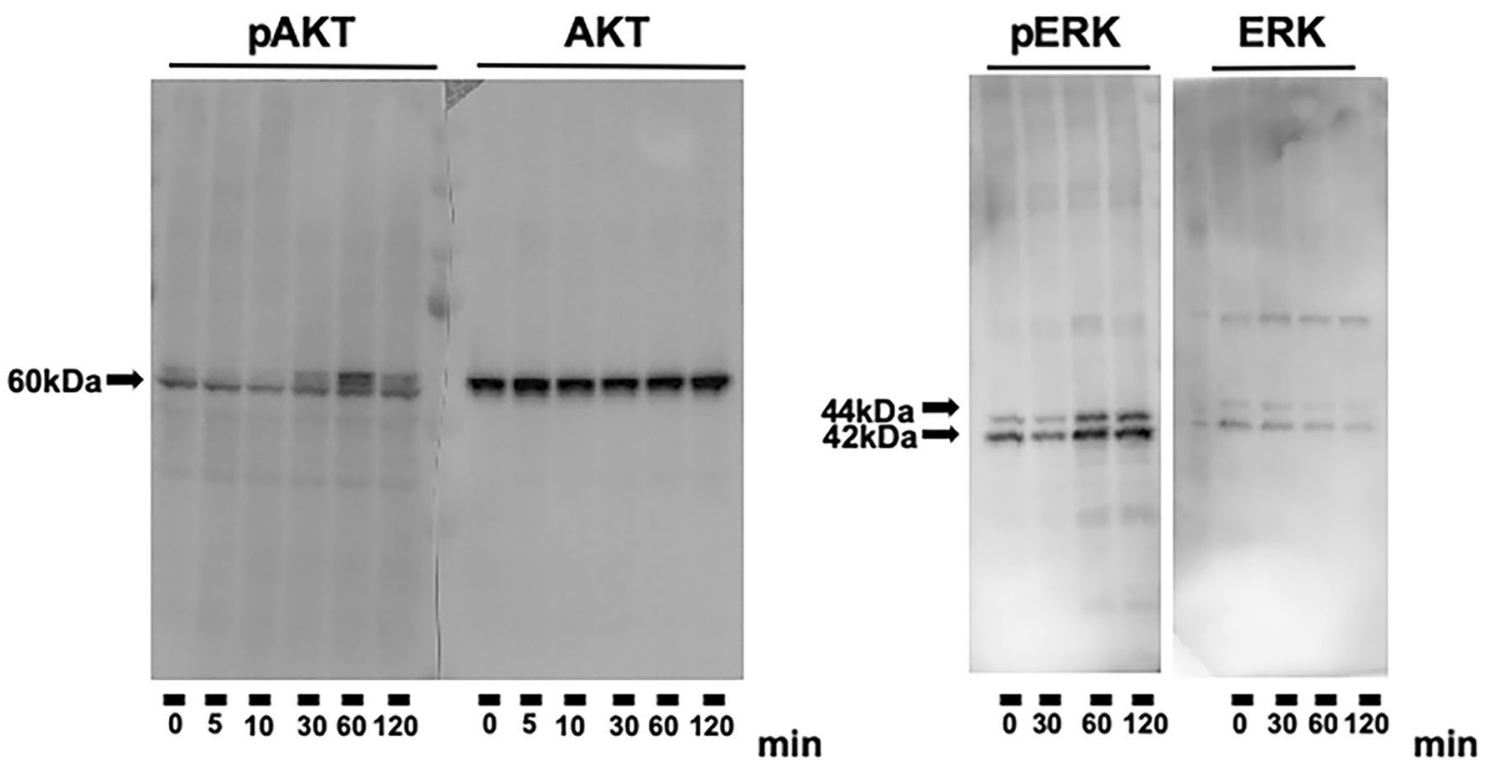

Fig. 3 Treatment with $100 \mu \mathrm{M}$ zonisamide for $60 \mathrm{~min}$ or $120 \mathrm{~min}$ induces phosphorylation of AKT and ERK1/2 in ND7/23 cells. The representative pictures of the western blot analysis are shown; similar findings are obtained by three experiments

Fig. 4 Zonisamide exhibits no significant effects on proliferation/survival of IFRS1; MTS assay. a Representative photomicrographs of control, forskolin, and zonisamide $100 \mu \mathrm{M}$-treated IFRS1 at Day 1. Scale bar $=100 \mathrm{~m}$. b Bar charts of the absorbance at $490 \mathrm{~nm}$ at Day 1 and Day 3 after treatment with $2 \mu \mathrm{M}$ forskolin (a positive control), $1 \mu \mathrm{M}, 10 \mu \mathrm{M}$, or $100 \mu \mathrm{M}$ zonisamide. Values represent means + SD from $7-8$ experiments; $* P<0.01$ (a)
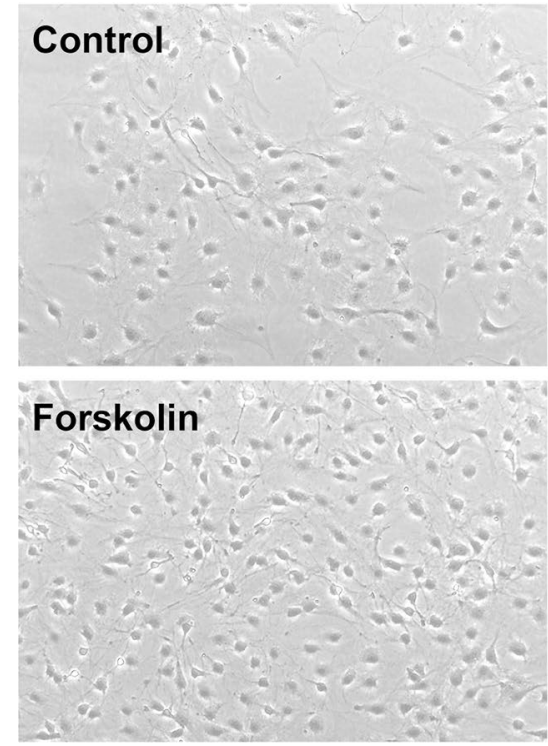

\section{Zonisamide $100 \mu \mathrm{M}$}

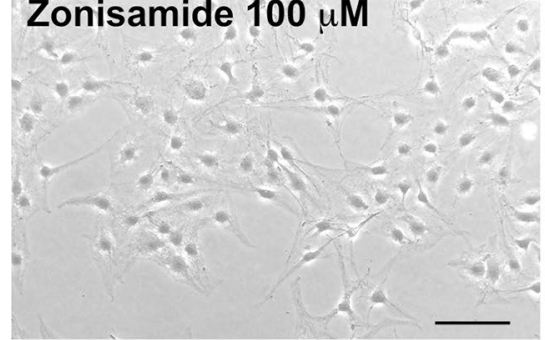

(b)

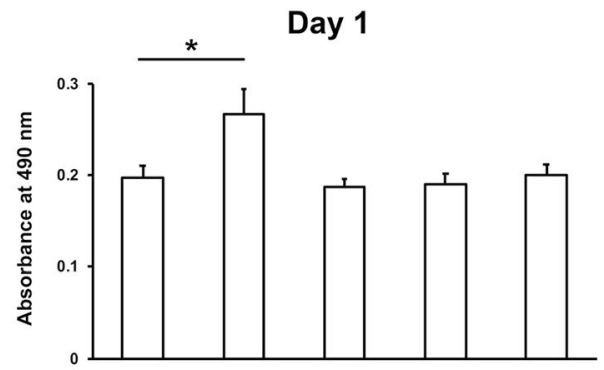

Day 3

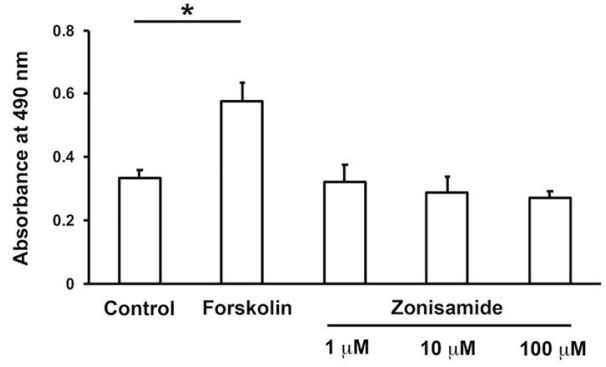


phosphorylation to protect motor neurons and NSC-34 cells from oxidative stress-induced injury and death (Yagi et al. 2015). Our current study focuses on the unsolved problems raised above, as well as possible cross talk between the PI3K and MAPK pathways and the downstream targets of these pathways responsible for zonisamide-induced neurite outgrowth.

\section{Zonisamide does not promote proliferation/survival or migration of IFRS1}

In contrast to its neurite outgrowth-promoting activity described above, zonisamide failed to enhance proliferation/survival (Fig. 4) or migration (Fig. 5) of IFRS1. These findings led us to speculate that zonisamide facilitates axonal regeneration through its direct actions on neurons rather than the stimulation of Schwann cell activity. However, we cannot deny the possibility that zonisamide potentiates synthesis and secretion of neurotrophic factors and cytokines in Schwann cells to augment neuroprotective system against axonal injury. We plan to explore that possibility by employing DNA microarray analysis, real-time RT-PCR analysis, and enzyme immunoassay (Niimi et al. 2018). The findings that zonisamide increased the reduced glutathione (GSH)

(a)

Day 0

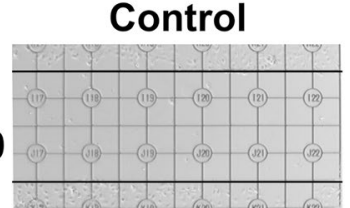

Day 3

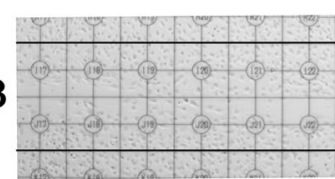

(b)

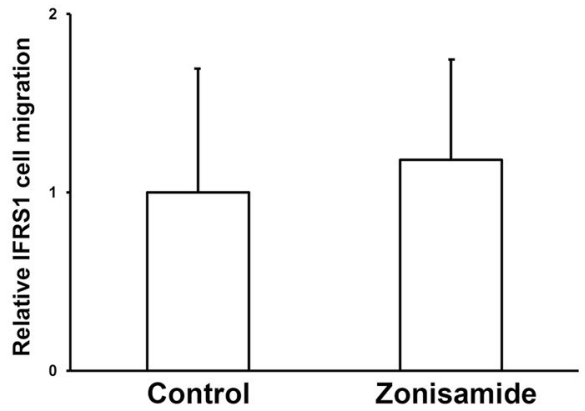

Fig. 5 Zonisamide exhibits no significant effects on migration of IFRS1; scratch wound assay. a Representative photomicrographs of control and zomisamide $(100 \mu \mathrm{M})$-treated IFRS1 at Day $0(2 \mathrm{~h}$ after scratch) and Day 3. Scratch was generated between the 2 thin black lines in each picture. Scale $b a r=100 \mu \mathrm{m}$. b Bar charts of the relative number of migrating cells in the presence or absence of $100 \mu \mathrm{M}$ zonisamide after 3 days of scratch generation. Values represent means + SD from five experiments level in astroglial cells, but not in dopaminergic neurons (Asanuma et al. 2010) suggests its beneficial effects on glial cells to protect the nervous system from oxidative stress and progressive neurodegeneration.

\section{Potential repositioning of zonisamide for peripheral nerve lesions}

The previous (Yagi et al. 2015) and present studies have demonstrated the neurotrophic activity of zonisamide on both motor and sensory neurons in the PNS and suggested its potential utility for peripheral nerve lesions. Because the effective zonisamide concentrations in these studies are $10-100 \mu \mathrm{M}$, ranging from those in sera of patients with Parkinson's disease (up to around $20 \mu \mathrm{M}$; Sumitomo Dainippon Pharma Co., Ltd., unpublished data) to those in sera of epilepsy patients (50-200 $\mu \mathrm{M})$ (Mimaki 1998), it seems fair to suppose that the research protocols have some clinical relevance. However, several limitations of our studies must be considered. First, there is a topographic difference in the site of neurite initiation and elongation; neurites sprout from neuronal cell bodies in vitro, whereas axonal regeneration occurs at the sites of injury in vivo. Second, individual neurons are isolated from other neurons and non-neuronal cells in vitro, and the effects of cellular interplay on axonal regeneration cannot be evaluated. To overcome these defects, we have established a three-dimensional culture system of ganglion explants in which adult peripheral ganglia with nerve fibers are embedded in collagen gel or Matrigel ${ }^{\circledR}$, and neurite outgrowth from transected nerve stump has been evaluated (Horie et al. 1994; Sango et al. 2017). Because of the maintenance of cell-to-cell interactions, it may be reasonable to consider that the explant culture mimics axonal regeneration in vivo better than the use of dissociated cells. Our preliminary study with the explant culture has suggested significant neurite outgrowth-promoting activity of zonisamide, which will be reported elsewhere.

To our knowledge, this is the first study to investigate the bioactivities of zonisamide on DRG neurons and Schwann cells. Although further analyses are needed to elucidate more precise action mechanisms of zonisamide, it is expected that zonisamide will be applicable for accelerating axonal regeneration with functional recovery after peripheral nerve injury and restoring diabetic and other peripheral neuropathies (Hord et al. 2003; Tanabe et al. 2008).

Acknowledgements We would like to thank Prof. Atsufumi Kawabata and Dr. Fumiko Sekiguchi for providing us ND7/23 cells, and Drs. Mari Suzuki, Hideji Yako, Naoko Niimi, and Tomoyo Akamine for helpful comments on this work.

Author contributions S.T. conducted cell culture, immunocytochemical analysis, and western blot analysis. K.S. designed the experiments, 
conducted cell culture and supervised the project. S.T. and K.S. drafted the manuscript.

\section{Compliance with ethical standards}

Conflict of interest With regard to the present study, the corresponding author received research funding and zonisamide from Sumitomo Dainippon Pharma Co., Ltd., Japan.

Open Access This article is licensed under a Creative Commons Attribution 4.0 International License, which permits use, sharing, adaptation, distribution and reproduction in any medium or format, as long as you give appropriate credit to the original author(s) and the source, provide a link to the Creative Commons licence, and indicate if changes were made. The images or other third party material in this article are included in the article's Creative Commons licence, unless indicated otherwise in a credit line to the material. If material is not included in the article's Creative Commons licence and your intended use is not permitted by statutory regulation or exceeds the permitted use, you will need to obtain permission directly from the copyright holder. To view a copy of this licence, visit http://creativecommons.org/licenses/by/4.0/.

\section{References}

Asanuma M, Miyazaki I, Diaz-Corrales FJ, Kimoto N, Kikkawa Y, Takeshima M, Miyoshi K, Murata M (2010) Neuroprotective effects of zonisamide target astrocyte. Ann Neurol 67:239-249

Chan KM, Gordon T, Zochodne DW, Power HA (2014) Improving peripheral nerve regeneration: from molecular mechanisms to potential therapeutic targets. Exp Neurol 261:826-835

Faroni A, Mobasseri SA, Kingham PJ, Reid AJ (2015) Peripheral nerve regeneration: experimental strategies and future perspectives. Adv Drug Deliv Rev 82-83:160-167

Hord AH, Denson DD, Chalfoun AG, Azevedo MI (2003) The effect of systemic zonisamide (Zonegran) on thermal hyperalgesia and mechanical allodynia in rats with an experimental mononeuropathy. Anesth Analg 96:1700-1706

Horie H, Kadoya T, Hikawa N, Sango K, Inoue H, Takeshita K, Asawa R, Hiroi T, Sato M, Yoshioka T, Ishikawa Y (2004) Oxidized galectin-1 stimulates macrophages to promote axonal regeneration in peripheral nerves after axotomy. J Neurosci 24:1873-1880

Inoue A, Ohnishi M, Fukutomi C, Kanoh M, Miyauchi M, Takata T, Tsuchiya D, Nishio H (2012) Protein kinase A-dependent substance $\mathrm{P}$ expression by pituitary adenylate cyclase-activating polypeptide in rat sensory neuronal cell line ND7/23 cells. J Mol Neurosci 48:541-549

Jain KK (2000) An assessment of zonisamide as an anti-epileptic drug. Expert Opin Pharmacother 1:1245-1260

Kawajiri S, Machida Y, Saiki S, Sato S, Hattori N (2010) Zonisamide reduces cell death in SH-SY5Y cells via an anti-apoptotic effect and by upregulating MnSOD. Neurosci Lett 481:88-91

Liang CC, Park AY, Guan JL (2007) In vitro scratch assay: a convenient and inexpensive method for analysis of cell migration in vitro. Nat Protoc 2:329-333

Matusica D, Fenech MP, Rogers ML, Rush RA (2008) Characterization and use of the NSC-34 cell line for study of neurotrophin receptor trafficking. J Neurosci Res. 86:553-565

Mimaki T (1998) Clinical pharmacology and therapeutic drug monitoring of zonisamide. Ther Drug Monit 20:593-597

Mitani K, Sekiguchi F, Maeda T, Tanaka Y, Yoshida S, Kawabata A (2016) The prostaglandin E2/EP4 receptor/cyclic AMP/T-type $\mathrm{Ca}(2+)$ channel pathway mediates neuritogenesis in sensory neuron-like ND7/23 cells. J Pharmacol Sci 130:177-180
Murata M, Hasegawa K, Kanazawa I, Fukasaka J, Kochi K, Shimazu R; Japan Zonisamide on PD Study Group (2015) Zonisamide improves wearing-off in Parkinson's disease: a randomized, double-blind study. Mov Disord 30:1343-5130

Murata M, Hasegawa K, Kanazawa I, Shirakura K, Kochi K, Shimazu R; Japan Zonisamide on PD Study Group (2016) Randomized placebo-controlled trial of zonisamide in patients with Parkinson's disease. Neurol Clin Neurosci 4:10-15

Niimi N, Yako H, Takaku S, Kato H, Matsumoto T, Nishito Y, Watabe K, Ogasawara S, Mizukami H, Yagihashi S, Chung SK, Sango K (2018) A spontaneously immortalized Schwann cell line from aldose reductase-deficient mice as a useful tool for studying polyol pathway and aldehyde metabolism. J Neurochem 144:710-722

Okada K, Tanaka H, Temporin K, Okamoto M, Kuroda Y, Moritomo H, Murase T, Yoshikawa H (2010) Methylcobalamin increases Erk1/2 and Akt activities through the methylation cycle and promotes nerve regeneration in a rat sciatic nerve injury model. Exp Neurol 222:191-203

Padhy BM, Gupta YK (2011) Drug repositioning: re-investigating existing drugs for new therapeutic indications. J Postgrad Med $57: 153-160$

Roloff F, Ziege S, Baumgärtner W, Wewetzer K, Bicker G (2013) Schwann cell-free adult canine olfactory ensheathing cell preparations from olfactory bulb and mucosa display differential migratory and neurite growth-promoting properties in vitro. BMC Neurosci 14:141

Saijilafu HEM, Liu CM, Jiao Z, Xu WL, Zhou FQ (2013) PI3K-GSK3 signalling regulates mammalian axon regeneration by inducing the expression of Smad1. Nat Commun 4:2690

Sango K, Yanagisawa H, Komuta Y, Si Y, Kawano H (2008) Neuroprotective properties of ciliary neurotrophic factor for cultured adult rat dorsal root ganglion neurons. Histochem Cell Biol 130:669-679

Sango K, Yanagisawa H, Kawakami E, Takaku S, Ajiki K, Watabe K (2011) Spontaneously immortalized Schwann cells from adult Fischer rat as a valuable tool for exploring neuron-Schwann cell interactions. J Neurosci Res 89:898-908

Sango K, Mizukami H, Horie H, Yagihashi S (2017) Impaired axonal regeneration in diabetes Perspective on the underlying mechanism from in vivo and in vitro experimental studies. Front Endocrinol $8: 12$

Satoh T, Nakatsuka D, Watanabe Y, Nagata I, Kikuchi H, Namura S (2000) Neuroprotection by MAPK/ERK kinase inhibition with U0126 against oxidative stress in a mouse neuronal cell line and rat primary cultured cortical neurons. Neurosci Lett 288:163-166

Takaku S, Yanagisawa H, Watabe K, Horie H, Kadoya T, Sakumi K, Nakabeppu Y, Poirier F, Sango K (2013) GDNF promotes neurite outgrowth and upregulates galectin-1 through the RET/PI3K signaling in cultured adult rat dorsal root ganglion neurons. Neurochem Int 62:330-339

Tanabe M, Murakami T, Ono H (2008) Zonisamide suppresses pain symptoms of formalin-induced inflammatory and streptozotocininduced diabetic neuropathy. J Pharmacol Sci 107:213-220

Tsukamoto M, Niimi N, Sango K, Takaku S, Kanazawa Y, Utsunomiya K (2015a) Neurotrophic and neuroprotective properties of exendin-4 in adult rat dorsal root ganglion neurons: involvement of insulin and RhoA. Histochem Cell Biol 144:249-259

Tsukamoto M, Sango K, Niimi N, Yanagisawa H, Watabe K, Utsunomiya K (2015b) Upregulation of galectin-3 in immortalized Schwann cells IFRS1 under diabetic conditions. Neurosci Res 92:80-85

Ulmann L, Rodeau JL, Danoux L, Contet-Audonneau JL, Pauly G, Schlichter R (2009) Dehydroepiandrosterone and neurotrophins favor axonal growth in a sensory neuron-keratinocyte coculture model. Neuroscience 159:514-525 
Wood JN, Bevan SJ, Coote PR, Dunn PM, Harmar A, Hogan P, Latchman DS, Morrison C, Rougon G, Theveniau M, Wheatley S (1990) Novel cell lines display properties of nociceptive sensory neurons. Proc Biol Sci 241:187-194

Yagi H, Ohkawara B, Nakashima H, Ito K, Tsushima M, Ishii H, Noto K, Ohta K, Masuda A, Imagama S, Ishiguro N, Ohno K (2015) Zonisamide enhances neurite elongation of primary motor neurons and facilitates peripheral nerve regeneration in vitro and in a mouse model. PLoS ONE 10:e0142786

Yamada H, Komiyama A, Suzuki K (1995) Schwann cell responses to forskolin and cyclic AMP analogues: comparative study of mouse and rat Schwann cells. Brain Res 681:97-104
Yin K, Baillie GJ, Vetter I (2016) Neuronal cell lines as model dorsal root ganglion neurons: a transcriptomic comparison. Mol Pain 12

Zigmond RE (2012) gp130 cytokines are positive signals triggering changes in gene expression and axon outgrowth in peripheral neurons following injury. Front Mol Neurosci 4:62

Publisher's Note Springer Nature remains neutral with regard to jurisdictional claims in published maps and institutional affiliations. 